\title{
FIRST PRINCIPLES CALCULATIONS FOR LITHIATED MANGANESE OXIDES
}

by
R. Prasad, R. Benedek, and M. M. Thackeray
*Chemical Technology Division, Argonne National Laboratory
and
J. M. Wills
Theoretical Division,

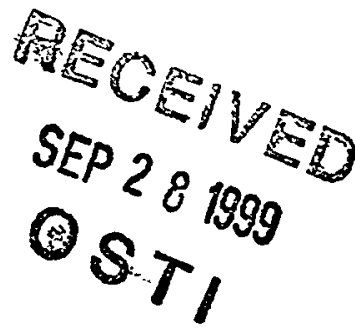
Los Alamos National Laboratory,
Los Alamos, New Mexico

and

L. H. Yang

Condensed Matter Physics Division, Lawrence Livermore National Laboratory

Livermore, $\mathrm{CA}$

The submitted manuscript has been created by the University of Chicago as Operator of Argonne National Laboratory ("Argonne") under Contract No. W-31-109-ENG-38 with the U.S. Department of

Energy. The U.S. Government retains for itself, and others acting on its behalf, a paid-up, nonexclusive, irrevocable worldwide license in said article to reproduce, prepare derivative works, distribute copies to the public, and perform publicly and display publicly, by or on behalf of the Government.

November 1998

To be published in the proceedings of the Materials Research Society Meeting held November 30 - December 4, 1998, Solid State Ionics Session. 


\section{DISCLAIMER}

This report was prepared as an account of work sponsored by an agency of the United States Government. Neither the United States Government nor any agency thereof, nor any of their employees, make any warranty, express or implied, or assumes any legal liability or responsibility for the accuracy, completeness, or usefulness of any information, apparatus, product, or process disclosed, or represents that its use would not infringe privately owned rights. Reference herein to any specific commercial product, process, or service by trade name, trademark, manufacturer, or otherwise does not necessarily constitute or imply its endorsement, recommendation, or favoring by the United States Government or any agency thereof. The views and opinions of authors expressed herein do not necessarily state or reflect those of the United States Government or any agency thereof. 


\section{DISCLAIMER}

Portions of this document may be illegible in electronic image products. Images are produced from the best available original document. 


\title{
FIRST PRINCIPLES CALCULATIONS FOR LITHIATED MANGANESE OXIDES
}

by

\author{
R. Prasad*, R. Benedek*, M. M. Thackeray*, J. M. Wills**, and L. H. Yang***
}

\begin{abstract}
First principles calculations within the local-spin-density-functional theory (LSDFF) framework are presented of densities of electronic states for $\mathrm{MnO}, \mathrm{LiMnO}_{2}$ in the monoclinic and orthorhombic structures, cubic $\mathrm{LiMn}_{2} \mathrm{O}_{4}$ spinel and $\lambda-\mathrm{MnO}_{2}$ (delithiated spinel), all in antiferromagnetic spin configurations. The changes in energy spectra as the Mn oxidation state varies between $2+$ and $4+$ are illustrated. Preliminary calculations for $\mathrm{Co}$-doped $\mathrm{LiMnO}_{2}$ are presented, and the destabilization of a monoclinic relative to a rhombohedral structure is discussed.
\end{abstract}

\section{INTRODUCTION}

First principles methods, particularly those based on local-density-functional theory (LDFT), are becoming a valuable tool for elucidating the structure and electrochemical properties of candidate cathode materials for $\mathrm{Li}$ insertion batteries. LDFT total energy calculations in conjunction with cluster expansions have recently been employed [1,2] to investigate phase stability and $\mathrm{Li}$ ordering in the layered oxide $\mathrm{Li}_{\mathbf{x}} \mathrm{CoO}_{2}$, currently used in commercial Li-ion batteries. Some of the present authors have recently performed LDFT calculations to investigate the structure of the two monoclinic phases of the vanadium gamma bronze, $\mathrm{Li}_{1+x} \mathrm{~V}_{3} \mathrm{O}_{8}$ [3], a material also under consideration as a possible cathode material. A

\footnotetext{
* Chemical Technology Division, Argonne National Laboratory

** Theoretical Division, Los Alamos National Laboratory, Los Alamos, New Mexico

*** Condensed Matter Physics Division, Lawrence Livermore National Laboratory
} 
great deal of experimental attention is currently being devoted to lithiated Mn oxides, owing to their practical advantages compared to the Co-based system. Improved theoretical understanding would benefit this effort.

A complication for $\mathrm{Mn}$ oxides is the strongly magnetic character of $\mathrm{Mn}$ ions. Although density functional theory calculations show that spin polarization has a small effect for $\mathrm{Li}_{\mathbf{x}} \mathrm{CoO}_{2}$ (total energy reduction of order $0.01 \mathrm{eV} /$ formula unit [1] relative to non-spin polarized calculations), which is most likely also the case for the vanadium $\gamma$-bronze [3], calculations for monoclinic $\mathrm{LiMnO}_{2}$ [4], show an energy reduction of several tenths of an $\mathrm{eV}$ per formula unit in spin-polarized treatments. Spin-polarization is therefore essential to obtain the most realistic description of Mn oxides. In recent work [5], Mishra and Ceder have performed an extensive first-principles study of manganese oxides in several crystal structures at different levels of lithiation both with and without spin polarization. They find that the relative stabilities and structures of the different phases are well described in calculations based on the generalized gradient approximation (GGA) [6], a formulation in which the LSDFT is augmented by gradient corrections. For all of the structures on which they made comparisons, antiferromagnetic configurations were favored over ferromagnetic configurations in the GGA-based calculations.

In this paper, densities of electronic states are presented for several standard Mn oxides in the antiferromagnetic state with $\mathrm{Mn}$ oxidation state between 2 and 4, which illustrate the behavior at various levels of filling of the majority spin $e$ band. Preliminary calculations for Codoped Mn dioxides are discussed, which are intended to elucidate the recent experimental finding [7] that such doping tends to stabilize the rhombohedral structure relative to the one with monoclinic symmetry. 


\section{METHOD}

Spin-polarized (LSDFT) as well as non spin-polarized (LDFT) calculations were performed with the full potential linearized muffin-tin orbital (FP-LMTO) code developed by J. M.. Wills [8]. Additional calculations were done with the plane wave pseudopotential code developed by Lin Yang [3], which is presently limited to LDFT. Troullier-Martins pseudopotentials are used in the plane wave calculations, with a cutoff energy of $70 \mathrm{Ry}$. The plane wave code is highly efficient numerically, and enables the treatment of large cells, up to at least several dozen atoms. The FP-LMTO code achieves high numerical precision in selfconsistent solutions to density functional theory, but is not as readily applied to large cells as the plane-wave formulation.

\section{RESULTS}

\section{Electronic Spectra}

Most Mn oxides undergo transitions to antiferromagnetic states at Neel temperatures that are below room temperature; disordered local moments with short range antiferromagnetic correlations exist above $\mathrm{T}_{\mathrm{N}}$. Figure 1 shows densities of electronic states calculated with the FP-LMTO method for antiferromagnetic $\mathrm{MnO}$ (rocksalt structure), $\mathrm{LiMnO}_{2}$ in the orthorhombic and monoclinic structures, $\mathrm{LiMn}_{2} \mathrm{O}_{4}$ in the (cubic) spinel structure and delithiated spinel $\left(\mathrm{Mn}_{2} \mathrm{O}_{4}\right)$. The calculations employ experimentally determined lattice constants and internal atomic coordinates. A small unit cell (two formula unit) approximant to the experimentally observed antiferromagnetic spin structure [9] is used for orthorhombic $\mathrm{LiMnO}_{2}$. In the case of monoclinic $\mathrm{LiMnO}_{2}[10]$, we used the AF3 configuration identified by Singh [4] as energetically 


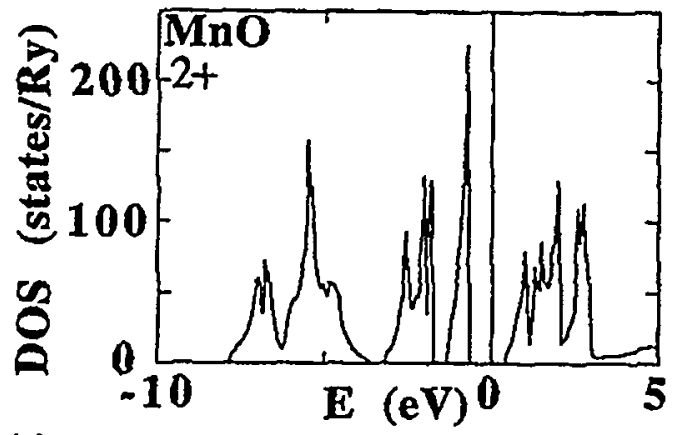

(a)

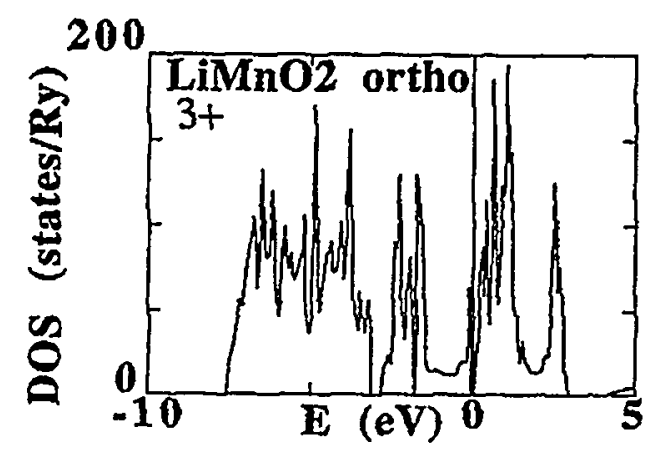

(c)

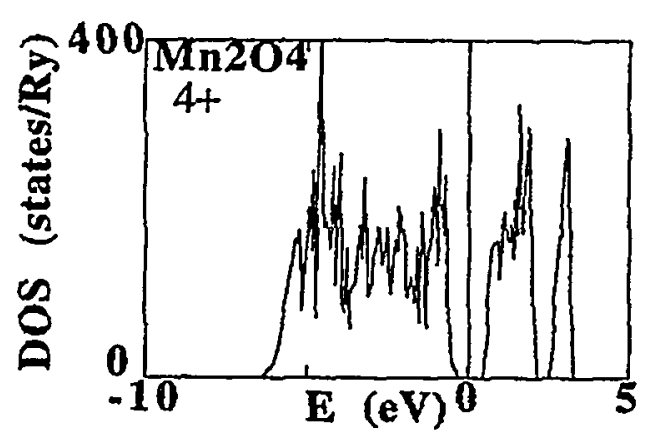

(e)

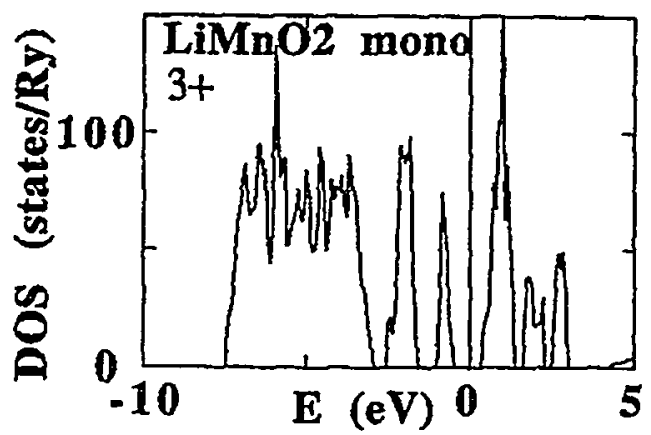

(b)

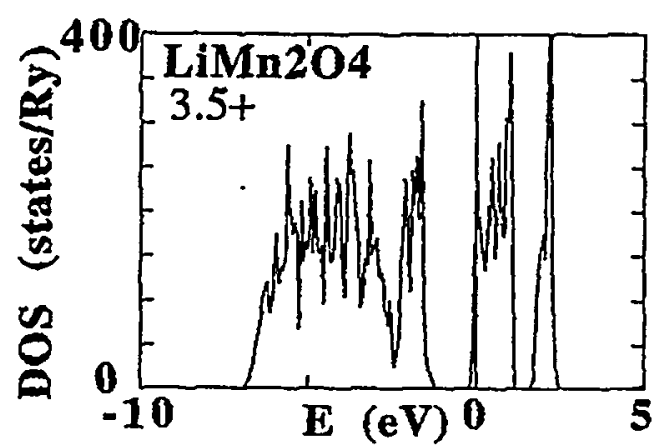

(d)

Fig. 1. Densities of electronic states for rocksalt $\mathrm{MnO}$ (a), $\mathrm{LiMnO2}$, in the monoclinic (b) and onhorhombic (c) structures, spinel (d) and delithiated spine! (e), calculated with the FP-LMTO method. Antiferromagnetic structures assumed in all cases. See text for discussion.

favorable. For spinel and delithiated spinel, we arbitrarily assigned two of the four Mn atoms per primitive unit cell as up spins and the other two as down spins. The pyrochlore- structure $\mathrm{Mn}$ sublattice of these systems is known to frustrate antiferromagnetic order, and results in large 
magnetic unit cells for spinel-derived structures $[11,12]$. The high temperature cubic atomic structure [13] was adopted for $\mathrm{LiMn}_{2} \mathrm{O}_{4}$ spinel; the recently measured $[12,14]$ atomic unit cell (tetragonal or orthorhombic) below the Verwey transition $\left[\mathrm{T}_{\mathrm{W}}-290 \mathrm{~K}\right]$ is complex. The zeroes on the abscissa axes in Fig. 1 denote Fermi levels. The lowest-lying states in each of the five systems correspond to the filled oxygen $2 p$ bands. The Mn $d$-bands straddle the Fermi level and are therefore only partially occupied. This band ordering is at variance with Hartree-Fock calculations [15], where the filled Mn- $d$ bands lie below the O-p bands, as a consequence of large spin-splitting $(-25 \mathrm{eV})$. The spin splitting of the Mn $d$-bands calculated within LSDFT is only about $3 \mathrm{eV}$. Photoemission experiments and configuration-interaction (CI) calculations [16], which show a $d$-band near the Fermi edge, appear to corroborate the band ordering predicted by LSDFT.

The majority spin $t_{2 \mathrm{~g}}$ band is filled for all the systems represented in Fig. 1. The central issue for the application of these materials to $\mathrm{Li}$ batteries is the structural instabilities that develop as the filling of the majority spin $e_{g}$ band is varied during the lithiation cycle. The majority spin $e_{\mathrm{g}}$ and $\mathrm{t}_{2 \mathrm{~g}}$ bands are both niieA in rocksalt $\mathrm{MnO}$, in which $\mathrm{Mn}$ adopts the maximum magnetic moment $5 \mu_{\mathrm{B}}$. (This system is included for comparison, but of course has no significance for batteries). The lithiated manganesedioxide systems, monoclinic and orthorhombic $\mathrm{LiMnO}_{2}$, correspond to a higher $\mathrm{Mn}$ oxidation state, 3+, and $e_{\mathrm{g}}$ is therefore only half filled. The Jahn-Teller distorted $\mathrm{Mn}-\mathrm{O}$ coordination octahedra in these systems split the occupied and unoccupied $e_{\mathrm{g}}$ bands and results in a gap (a small one in the case of the orthorhombic structure, but we have not employed the full antiferromagnetic unit cell for this system [9]). If $\mathrm{LiMn}_{2} \mathrm{O}_{4}$ spinel is considered to possess an equal mixture of $3+$ and $4+\mathrm{Mn}$ ions, 
only half of those ions prefer a JT-distorted environment, whereas all Mn environments are equivalent in the assumed cubic structure. It is possible that LSDFT calculations for the low temperature antiferromagnetic structure [12,14], which are not presently computationally feasible because of its large unit cell, would yield a gap; however, residual disorder of the $\mathrm{Mn}$ charge states appears to persist in this system down to the lowest temperatures [12]. In the case of the fully delithiated spinel $\mathrm{Mn}_{2} \mathrm{O}_{4}\left(\lambda-\mathrm{MnO}_{2}\right)$, the $e_{\mathrm{g}}$ level is completely empty, and a gap is observed between it and the filled $t_{2 \mathrm{~g}}$

Despite the energy gaps that appear in Fig. 1, these systems should not be regarded as band insulators. The observed polaronic character of electron transport indicates that electron localization effects that are beyond the scope of LSDFT are significant for Mn oxides. Nevertheless, the work of Mishra and Ceder [5] provides evidence that density functional theory can yield realistic total energies and relative stabilities of competitive Mn oxides structures.

\section{Jahn- Teller Distortion}

Technically the most difficult regime for first principles calculations is that for which both $3+$ and $4+\mathrm{Mn}$ oxidation states are present, as for example in $\mathrm{Li}_{x} \mathrm{Mn}_{2} \mathrm{O}_{4}(0<x<1)$. In such systems, a disordered arrangement of if distorted octahedra typically occurs in conjunction with a frustrated antiferromagnetic spin configuration. In several spinel-based Systems, notably those of the Li-Mn-O ternary system, static JT distortions are observed when the mean Mn oxidation state is below about 3.5 [17]. An additional complication is the order-disorder transitions on the Li sublattice, which have been addressed theoretically in the context of Co oxides $[1,2]$, but not Mn oxides. The treatment of all these features simultaneously, i.e., the JT distortions, the 
antiferromagnetic structure, and the $\mathrm{Li}$ configuration at arbitrary $\mathrm{x}$, pose a severe challenge to theory.

The "endpoints" with mean $\mathrm{Mn}$ valence very close to either 3 or 4 are relatively more tractable from the standpoint of first principles calculations than the mixed valent regime. In the case of $\mathrm{Mn}(3+)$, e.g. in monoclinic [4] and orthorhombic [9] $\mathrm{LiMnO}_{2}$, the JT-distortions are uniform throughout the crystal, and the antiferromagnetic structure is unfrustrated, which lead to a relatively small unit cell. In the case of Mn 4t, although the magnetic unit cell may be frustrated (e.g., in $\lambda-\mathrm{MnO}_{2}$ [11]), JT distortions are absent, and even non-spin-polarized LDFT calculations may be adequate for some purposes. Thus, we find excellent agreement between calculated internal coordinates and experiment in non-spin-polarized pseudopotential LDFT calculations for $\alpha-\mathrm{Mn}_{2} \mathrm{O}_{4}$ (hollandite), as well as for the electrochemical potential of an inserted $\mathrm{Li}$ atom; that work will be presented elsewhere.

\section{Co donin Q of lavered $\mathrm{LiMnO}_{2}$}

The mixed valent regime is crucial to the operation of Li batteries. A practical difficulty is the limited cycle lifetime of Mn-based systems resulting from JT-induced structural transformations [17], which has motivated the exploration of strategies to suppress the JT distortion. The recent discovery [7] that the rhombohedral $\left(\alpha-\mathrm{NaFeO}_{2}\right)$ structure of $\mathrm{LiMnO}_{2}$ is stabilized with respect to the (JT distorted) monoclinic form by substitution of only $10 \%$ Co suggests that JT-distortion suppression may be possible even at mean Mn valences well below 3.5. 
The detailed mechanism for this suppression has yet to be established. Experience with the somewhat analogous JT system perovskite $\mathrm{LaMnO}_{3}$ may be relevant in this context: (i) only $20 \%$ divalent doping ( $\mathrm{Sr}$ ) on the La sublattice suppresses the cooperative JT phase transition [18], and (ii) Co substituted on the Mn sublattice is divalent [19]. These results suggest that modest levels of divalent cobalt in $\mathrm{Li}\left(\mathrm{Co}_{\mathrm{x}} \mathrm{Mn}_{1-\mathrm{x}}\right) \mathrm{O}_{2}$ might be sufficient to suppress the JT distortion.

We have begun FP-LMTO calculations on $\mathrm{Li}\left(\mathrm{Co}_{\mathrm{x}} \mathrm{Mn}_{1-\mathrm{x}}\right) \mathrm{O}_{2}$ to elucidate the effect of Co doping. Both the monoclinic and the rhombohedral structures were considered, and the AF3 [4] anti ferromagnetic spin configuration was assumed. The dopant calculations employ one Co atom in a 16 atom cell $(x=0.25)$. Calculations for 8-atom undoped cells $(x=0.0)$ were also performed. For the undoped case, non-spin-polarized and ferromagnetic as well as antiferromagnetic configurations were investigated. These calculations (as well as those presented above for the electronic densities of states) are based on the LSDFT, and do not include the GGA $[5,6]$. Our calculations have thus far been restricted to the experimental atomic coordinates [10] for the monoclinic structure and the LDFT-predicted [20] atomic coordinates for rhombohedral $\mathrm{LiMnO}_{2}$, and lattice relaxation was not considered either in the doped or the undoped cases.

The total energy difference between the rhombohedral and monoclinic $\mathrm{Li}\left(\mathrm{Co}_{\mathrm{x}} \mathrm{Mn}_{1-\mathrm{x}}\right) \mathrm{O}_{2}$ structures in the undoped case $\Delta E_{r-m}(x=0)=0.18 \mathrm{eV} /$ formula unit, consistent with the known stability of the monoclinic structure [10]. We note, however, that Mishra and Ceder [5] found the ferromagnetic rhombohedral structure to be more stable than the antiferromagnetic monoclinic structure at the LSDFT level and only at the GGA level was the latter more stable. 
We expect, however, that by comparing doped and undoped cases at the same level of approximation that valid trends may be discerned.

In the case of the doped system we find $\left.\Delta E_{r-m}(x=0.25)=0.20\right) .20-\mathrm{eV} /$ formula unit. If we take at face value both this result and that for the undoped system, it would suggest that $\Delta E_{r-m}(x \sim 0.1)=0.0$, consistent with the experimental observation of rhombohedral structure stabilization at $10 \%$ doping [7]. Analysis of the spin up and spin down integrated charge densities in the muffin-tins of the $\mathrm{Mn}$ atoms; however, show them (in both monoclinic and rhombohedral structures) to be unchanged by the Co dopant, which suggests that no charge transfer takes place and the oxidation state of Co is $3+$ rather than $2+$. We suspect, however, that the neglect of lattice relaxation may be giving misleading results. S ince the JT environment of monoclinic $\mathrm{LiMnO}_{2}$ is unfavorable for $\mathrm{Co}$, we expect that the coordinated oxygens may relax appreciably, making it favorable for neighboring $\mathrm{Mn}$ to reduce the Co oxidation state to $2+$, and and correspondingly increase the oxidation state of Mn above 3+. This conjecture, of course, must be confirmed or refuted by explicit calculation, which is now in progress.

\section{CONCLUSION}

First principles calculations for lithiated Mn oxides are most difficult in the mixed valent regime, which is the regime of greatest practical interest. A direction that we are currently pursuing is to apply such calculations to gain an understanding of how dopants may suppress the cooperative JT phase transitions that affect cycle life of $\mathrm{Mn}$-based electrodes in Li batteries.

\section{ACKNOWLEDGMENTS}


This work was supported at ANL by the U.S. Department of Energy, Office of Basic Energy Sciences. L.Y. is supported at LLNL by the U.S. Department of Energy under contract no. W-7405-ENG-48. Most of the computational work was performed at the National Energy Research Supercomputer Center.

\section{REFERENCES}

1. A. Van der Ven, M. K. Aydinol, G. Ceder, G. Kresse, and J. Hafner, Phys. Rev. B 58, 2975 (1998).

2. C. Wolverton and A. Zunger, Phys. Rev. Lett. 81, 606 (1998).

3. R. Benedek, M. M. Thackeray, and L. H. Yang, Phys. Rev. B 56, 10708 (1997);

Materials Research Society Symposium Proceedings Vol.496, 115 (1998).

4. D. J. Singh, Phys. Rev. B 55, 309 (1997).

5. $\quad$ S. K. Mishra and $U$. Ceder, preprint, 1998.

6. J. P. Perdew, J. A. Chevary, S. H. Vosko, K. A. Jackson, M. R. Pederson, D. J. Singh, and C. Fiolhais, Phys. Rev. B 46, 6671(1992).

7. A. R. Armstrong, R. Gitzendanner, A. D. Robertson, and P.O. Bruce, Chem. Commun.17, 1833 (1998).

8. J. M. Wills, unpublished.

9. J.E. Greedan, N. P. Raju and I. J. Davidson, J. Solid State Chem. 128, 209 (1997).

10. R. Armstrong and P.O. Bruce, Nature 381, 499 (1996); F. Capitaine, P. Uravereau, and C. Delmas, Solid State lonics 89, 197 (1996).

11. J. E. Greedan, N. P. Raju, A. S. Wills, C. Morin, J. Britten, H. Dabkowska, and S. M. Shaw, Chem. Mater. 10, 3058 (1998).

12. A. S. Wills, N. P. Raju, and J. E. Greedan, preprint 1998.

13. A. Yamada and M. Tanaka, Materials Research Bulletin, 30,715 (1995).

14. J. Rodriguez-Carvajal, U. Rousse, C. Masquelier, and M. Hervieu, Phys. Rev. Lett., in press (1998). 
15. W. C. Mackrodt, and E.A. Williamson, Phil. Mag. B 77, 1077 (1998).

16. R. Lad and V. E. Henrich, Phys. Rev. B 38, 10860 (1988).

17. M. M. Thackeray, Progress in Solid State Chem. 25, 1(1997).

18. B. A. A. Ellemans, B. van Laar, K. R. van der Veer, and B. 0. Loopstra, J. Solid State Chem. 3, 328 (1971).

19. J.-H. Park, S.-W. Cheong, and C. T. Chen, Phys. Rev. B 55, 11072 (1997).

20. M. K. Aydinol, A. F. Kohan, U. Ceder, K. Cho and 3. Joannopoulos, Phys. Rev. B 56, 1354 (1997). 